\title{
The role of miRNAs targeting K-ras and APC genes in colorectal cancer
}

\author{
Yilmaz $\mathrm{N}^{1,2}$, Yilmaz $\mathrm{U}^{1,3}$, Tanbek $\mathrm{K}^{3,4}$, Arikan $\mathrm{S}^{5}$, Aksakal $\mathrm{N}^{6}$, Zeybek $\mathrm{U}^{1}$, Ergen $\mathrm{A}^{1}$ \\ Department of Molecular Medicine, Aziz Sancar Institute of Experimental Medicine, Istanbul University, \\ Istanbul, Turkey. aergen@istanbul.edu.tr
}

\begin{abstract}
OBJECTIVES: The occurrence of abnormal expression patterns in different types of cancer suggests that micro RNAs (miRNAs) may play an important role in tumorigenesis. The aim of this study was to examine the expression levels of miRNAs known to be associated with the regulation of the expression levels of the APC and K-ras, which are important in the development of colorectal cancer (CRC).

METHODS: The expression levels of miR-27, miR-663, miR-217, miR-181d, APC and K-ras in the serum, tumor and adjacent tumor-free (healthy) tissues of the patients and serum of the healthy controls were investigated with qRT-PCR.

RESULTS: Expression levels of miR-217, mR-181d, miR-663, miR-27 and K-ras were found to be higher in CRC tissues than in adjacent tumor-free tissues of the patients. In patient serum samples, miR-663 levels were statistically more elevated than in controls. In patient tumor tissues, miR-217, miR-181d and miR-27 expressions were found to be higher.

CONCLUSIONS: Increased miR-181d and miR-217 expression levels are associated with increased K-ras expression in the tumor tissues, and the expression of K-ras, which takes part as an oncogene in the CRC development, might be regulated by these miRNAs (Tab. 4, Ref. 33). Text in PDF www.elis.sk KEYWORDS: colorectal, cancer, miRNA, APC, K-ras.
\end{abstract}

\section{Introduction}

Cancers developed due to the cumulative accumulation of genetic and epigenetic changes occurring in the normal colonic mucosa are called colorectal cancer (CRC). Mutations occur in the APC gene known to play an important role in cell cycle control, apoptosis, migration and adhesion, thus causing some regions of the APC protein in the colon epithelial cells not to be encoded, which results in problems in the mechanisms regulating cell growth and division $(1,2)$. The APC gene mutation, the earliest identified mutation in the adenoma-carcinoma process, is thought to be an initiator in adenoma formation and is observed in $98 \%$ of cases of CRC (3-5).

The K-ras mutation occurs immediately after the APC gene mutation in the adenoma to carcinoma process and is thought to increase the GTPase activity, which leads to an increase in cel-

${ }^{1}$ Department of Molecular Medicine, Aziz Sancar Institute of Experimental Medicine, Istanbul University, Istanbul, Turkey, ${ }^{2}$ Department of Anatomy, Faculty of Medicine, Inonu University, Malatya, Turkey, ${ }^{3}$ Department of Physiology, Faculty of Medicine, Inonu University, Malatya, Turkey, ${ }^{4}$ Department of Biochemistry, Faculty of Medicine, Inonu University, Malatya, Turkey, ${ }^{5}$ Department of General Surgery, Istanbul Education and Research Hospital, Istanbul, Turkey, and ${ }^{6}$ Department of General Surgery, Istanbul Faculty of Medicine, Istanbul University, Istanbul, Turkey

Address for correspondence: A. Ergen, Prof, MD, Department of Molecular Medicine, Aziz Sancar Institute of Experimental Medicine, Istanbul University, Vakif Gureba Cad., Sehremini.Fatih 34093 Istanbul, Turkey. Phone: +90.2124142000, Fax: + 90.2125324171

Acknowledgement: The present work was supported by the Research Fund of Istanbul University (Project No. 20707). lular proliferation and malignant transformation in CRC development $(6,7)$.

MicroRNAs (miRNAs) are single-stranded, 18-24 nucleotides long, RNAs that do not encode proteins and are naturally produced in cells (8). Abnormal expression patterns of miRNAs that play a role in regulating the expression of genes that control cellular functions such as proliferation, differentiation and apoptosis in different types of cancer indicate that they take an important part in tumorigenesis (9). The demonstration of different expression patterns between the tumor and normal tissues suggests that there may be a direct relationship between miRNAs and cancer, and that they may have a potential as biomarkers in the diagnosis, classification and treatment of cancer $(10,11)$.

The aim of this study was to examine the expression levels of miR-27, miR-663, miR-217, miR-181d,APC and K-ras in tumor and adjacent tumor-free (healthy) tissue samples of individuals with CRC. Expression levels of miR-27, miR-663, miR-217, and miR$181 \mathrm{~d}$ between tumor tissue and serum samples of CRC patients as well as between the serum samples of CRC patients and healthy controls were examined for the purpose of contributing to the development of new miRNA-based methods in the diagnosis and treatment of CRC.

\section{Materials and methods}

\section{Study Population}

CRC in the Department of General Surgery, Istanbul Faculty of Medicine, Istanbul University and 50 healthy indivi- 
duals with no personal or familial cancer history were included in this study.

Tumor tissue and adjacent tumor-free (healthy) tissue samples in cryogenic tubes were collected from the CRC patients as well as peripheral blood samples before the operation. Peripheral blood samples only were taken from healthy subjects defined as the control group. The serum was separated from all the blood samples with a centrifuge. This study was conducted with the approval of the Ethical Committee of the Istanbul Faculty of Medicine, Istanbul University $(874 / 2015)$.

\section{Total RNA and miRNA isolations}

The RNA isolation from the tumor tissue and the surrounding normal tissue were performed using Qiagen RNeasy Plus Mini Kit and miRNA isolation using Qiagen miRNeasy Mini Kit. MiRNA isolations from the serum samples of the healthy controls were done using Qiagen miRNeasy Serum/Plasma Kit. All procedures were performed according to the manufacturer's directions.

\section{cDNA Synthesis from the total RNA and miRNA samples}

The cDNA synthesis was performed using AccuScript High Fidelity 1 st Strand cDNA Synthesis Kit from the total RNA samples and from miRNA samples using miRNA 1st-Strand cDNA Synthesis Kit according to the manufacturer's directions.

\section{Quantitative Real-Time PCR Analyses}

Quantitative real-time-PCR analyses were performed using Agilent Mx3005P. The gene expression analyses of APC, K-ras and GAPDH (as control) were done utilizing RT2 QPCR Primer Assay and SYBR Green QPCR Master Mix. Expression levels of miR-27, miR-181d, miR-217 and miR-663 were determined using miScript Primer Assay and miRNA QPCR Master Mix. Relative gene expressions were calculated by $2^{-\Delta \triangle \mathrm{CT}}$, relative to the GAPDH and RNU6 controls.

\section{Statistical analyses}

For statistical analysis, SPSS (Statistical Package for Social Sciences for Windows) program 21.0 was utilized and it was considered significant when $p$ value was below 0.05 . The normal distribution of variables was assessed by Kolmogorov-SmirnovShapiro Wilk tests. The Student's t-test was used to compare the values of the individuals with normal distribution fit. Statistics were given as mean \pm standard deviation and median.

\section{Results}

Demographic and clinical features of the patient and control groups are given in Table 1. Expression levels of miR-217, miR$181 \mathrm{~d}$, miR-663, miR-27, APC and K-ras in the tissue samples of the patient group were evaluated. In tissue samples, miR-217, miR-181d, miR-663, miR-27 and K-ras expressions were found statistically higher than in the adjacent tumor-free (healthy) tissues, while APC was higher in adjacent tumor-free (healthy) tissue ( $p=0.007 ; 95 \%$ confidence interval $(\mathrm{CI})=0.47-2.95)$. No statistically significant elevation was found regarding mir-217 expression $(\mathrm{p}=0.05)$ (Tab. 2).
Tab. 1. Demographic and clinical features of the patient and control groups.

\begin{tabular}{|c|c|}
\hline$\overline{\text { Age (Year) (Patient Group) }}$ & $57.30 \pm 21.56$ \\
\hline Age (Year) (Control Group) & $56.30 \pm 19.56$ \\
\hline Sex (F/M) (Patient Group) & $20 / 27$ \\
\hline Sex (F/M) (Control Group) & $17 / 33$ \\
\hline Tumor stage & $\begin{array}{ll}\text { T1 } & 6.7 \% \\
\text { T2 } & 6.7 \% \\
\text { T3 } & 43.3 \% \\
\text { T4 } & 43.3 \%\end{array}$ \\
\hline Lymph node involvement & $\begin{array}{ll}\text { N0 } & 35.5 \% \\
\text { N1 } & 35.5 \% \\
\text { N2 } & 29 \% \\
\end{array}$ \\
\hline Presence of metastasis & $54.8 \%$ \\
\hline Presence of perineural invasion & $44 \%$ \\
\hline Differentiation & $\begin{aligned} & \text { poorly } 29 \% \\
& \text { well } 25.8 \% \\
& \text { moderately } 45.2 \% \\
&\end{aligned}$ \\
\hline Tumor localization & $\begin{aligned} & \text { sigmoid colon } 43.8 \% \\
& \text { rectum } 18.8 \% \\
& \text { right colon } 21.9 \% \\
& \text { left colon } 3.1 \% \\
& \text { rectosigmoid } 9.4 \% \\
& \text { splenic flexure } 3.1 \% \\
&\end{aligned}$ \\
\hline
\end{tabular}

Tab. 2. Expression levels of miR-217, miR-181d, miR-663, miR-27, APC and K-ras in the tumor and adjacent tumor-free (healthy) tissues.

\begin{tabular}{lcccc}
\hline $\begin{array}{l}\text { miRNAs } \\
\text { and genes }\end{array}$ & $2^{-\Delta \Delta C \mathrm{~T}}$ (min-max) & $\begin{array}{c}\text { Fold } \\
\text { change }\end{array}$ & $\mathrm{p}$ & $\begin{array}{c}95 \% \text { Confidence } \\
\text { interval }\end{array}$ \\
\hline miR-217 & $1.17(-6.47-9.41)$ & 8.45 & 0.05 & $(-0.04-2.38)$ \\
miR-181d & $0.76(-7.88-7.24)$ & 9.18 & 0.10 & $(-0.71-1.84)$ \\
miR-663 & $-0.44(-8.86-6.59)$ & 20.31 & 0.39 & $(-1.38-0.54)$ \\
miR-27 & $0.33(-7.58-5.53)$ & 10.21 & 0.40 & $(-0.48-1.119)$ \\
APC & $1.53(-4.15-7.87)$ & 1.58 & $0.007^{*}$ & $(0.47-2.95)$ \\
K-ras & $0.50(-7.07-16.48)$ & 16.32 & 0.94 & $(-1.75-1.87)$ \\
\hline
\end{tabular}

When the miRNA expressions were compared between the patient and control group serum samples, miR-663 was found to be statistically higher in the patient group $(\mathrm{p}=0.02 ; 95 \% \mathrm{CI}=$ 0.16-2.14) (Tab. 3).

Expressions of miR-217, miR-181d, miR-663 and miR-27 in the serum and tissue samples of the patients were evaluated; all miRNAs, beside miR-663, were observed to be elevated in the tissues (Tab. 4).

No statistical significance was observed when miR-217, miR181d, miR-663, miR-27, APC and K-ras expression levels were examined in terms of the presence of metastasis, node involvement and tumor stage in the patient tumor samples.

\section{Discussion}

miRNAs are known to take part in important cellular events such as cell proliferation, apoptosis and cancer development (12, 13). It is known that a large number of miRNAs play a role in the pathogenesis of CRC and studies are conducted to clarify the role of new miRNAs in this disease (14).

In this study, the role of miR-27, miR-663, miR-181d and miR-217 in CRC were investigated. These miRNAs, which have been studied in many different types of cancer, have been studied 
Tab. 3. Comparison of expression levels of miR-217, miR-181d, miR663 and miR-27 in serum samples of patient and control groups.

\begin{tabular}{lcccc}
\hline miRNAs & $2^{-\triangle \Delta C T}(\min -\max )$ & $\begin{array}{c}\text { Fold } \\
\text { change }\end{array}$ & $\mathrm{p}$ & $\begin{array}{c}95 \% \text { Confidence } \\
\text { interval }\end{array}$ \\
\hline miR-217 & $1.26(-5.72-10.56)$ & 11.80 & 0.38 & $(-1.71-4.23)$ \\
miR-181d & $0.80(-10.5-11.56)$ & 43.22 & 0.11 & $(-0.26-2.48)$ \\
miR-663 & $1.05(-6.57-9.11)$ & 4.85 & $0.02^{*}$ & $(0.16-2.14)$ \\
miR-27 & $-0.14(-13.37-14.17)$ & 44.19 & 0.86 & $(-1.82-1.53)$ \\
\hline
\end{tabular}

Tab. 4. Expression levels of miR-217, miR-181d, miR-663 and miR-27 among patient tissue and serum samples.

\begin{tabular}{lcccc}
\hline miRNAs & Patient Sample & $\Delta \mathrm{CT} \pm \mathrm{SD}$ & $\mathrm{p}$ & $\begin{array}{c}95 \% \text { Confidence } \\
\text { interval }\end{array}$ \\
\hline miR-217 & $\begin{array}{c}\text { tumor tissue } \\
\text { serum }\end{array}$ & $\begin{array}{r}5.55 \pm 3.10 \\
0.98 \pm 4.07\end{array}$ & 0.004 & $(1.71-7.41)$ \\
\hline miR-181d & $\begin{array}{c}\text { tumor tissue } \\
\text { serum }\end{array}$ & $\begin{array}{c}4.95 \pm 2.47 \\
-1.23 \pm 3.29\end{array}$ & 0.001 & $(4.85-7.51)$ \\
\hline miR-663 & $\begin{array}{c}\text { tumor tissue } \\
\text { serum }\end{array}$ & $\begin{array}{c}-1.56 \pm 2.69 \\
-0.18 \pm 2.82\end{array}$ & 0.02 & $(-2.53-0.22)$ \\
\hline miR-27 & tumor tissue & $3.00 \pm 1.97$ & 0.001 & $(6.13-9.12)$ \\
\hline
\end{tabular}

SD - Standard deviation

for their tumor suppressor or oncogenic properties in CRC in different samples of the subjects included.

In another study, it was shown that in addition to inhibiting cell migration and invasion, miR-27a suppresses cell proliferation and induces apoptosis in renal cell carcinoma. Therefore, it was said that miR-27a plays a role of a tumor suppressor in renal cell carcinoma (15). Furthermore, in another study, miR-27 expression has been shown to be 10 times higher in endometrial cancer tissues compared to its expression in normal endometrium (16). Similarly, in our study, it was also observed that the colorectal cancer patients had an increased expression of miR-27 in the tumor compared to its expression in the adjacent tumor-free (healthy) tissue. When the miR-27 expressions between serum samples and tumor tissues of the CRC patients were compared, we found that miR-27 expression was higher in the tumor tissues. However, when serum samples of the healthy individuals and patients were compared, we observed no difference regarding miR-27 expressions. Thus, we believe that the increase in miR-27 expression may play a role in tumor development in colorectal cancer.

In studies conducted on the effect of miR-663 on nasopharyngeal carcinoma, the serum level of miR-663 was found to be higher in patients than in the healthy controls $(17,18)$. The levels of miR-663 were also shown to be elevated in lung cancer patients (19). Wang et al have found that miR-663 expression was significantly increased as compared to healthy controls. Also, they have found a significant association of miR-663 expression with tumor differentiation, invasion, lymph node metastasis, and TNM stage (20). When we examined the expression of miR-663 in tumor and tumor-free tissue samples of the CRC patients, we observed increased expression levels in the tumor samples. In addition, when the miR-663 expression levels were compared between the serum samples of the healthy controls and patients, they were found to be elevated in the patient samples. It was also found that serum samples of the CRC patients showed a higher expression of miR-663 than the tumor samples.
Based on our findings, we are of the opinion that miR-663 may play a role as an oncogenic miRNA in the development of colorectal cancer.

In present study, APC gene expressions were also assessed in normal tissue and tumor tissue samples of the CRC patients, and it was observed that APC expression was statistically elevated in the normal tissues as compared to its expression in the tumor tissues. In many studies it has been confirmed that the APC gene is a direct target of miR-27 and miR-663 (21-24). Based on our findings, we think that increased expression levels of miR-27 and miR-663 in the tumor tissue are also related to the decreased APC gene expression in the tumor tissue. We suggest that the expression of the APC gene, which plays a role as a tumor suppressor in the development of CRC, is suppressed by these miRNAs and unable to function fully. It can be said that miR-27 and miR-663, which suppress the expression of APC, i.e. the tumor suppressor gene, can function as oncogenic miRNAs in the development of CRC.

It was shown that miR-181d was upregulated in CRC tissues compared to the controls. Also, increased expression of miR-181d has been found to promote cell proliferation, migration, and invasion (25). Huang et al have demonstrated that expression of miR$181 \mathrm{~d}$ was correlated with tumor localization, TNM stage and local recurrence. They have also suggested that the expression of miR$181 \mathrm{~d}$ may be an independent prognostic factor for CRC (26). In our study, we examined the miR-181d expression in tumors and normal tissue samples of the CRC patients and found that it was increased in the tumor tissue compared to normal tissue. When miR-181d expressions between the serum samples and tumor tissues were compared, the expression of miR-181d in tumor tissue was observed to be higher than that in serum samples. Furthermore, no difference was found between patients and healthy controls regarding serum miR-181d levels. A higher expression of miR-181d was observed in the serum samples of the patients with advanced cancer, while no difference was observed between the tissue and serum samples of the patients in terms of miR-181d expression according to the presence of metastasis and node involvement. We suggest that miR$181 \mathrm{~d}$ may play a role in cancer development, considering its higher expression in CRC patient tumor tissues than in normal tissues.

Zhang et al reported that miR-217 expression in breast cancer tissues was markedly upregulated when compared to normal breast tissues (27). Moreover, Vychytilova-Faltejskova et al, found that miR-217 levels in their study were lower in pancreatic ductal adenocarcinomas than in healthy tissues and chronic pancreatitis (28). In our study, when the miR-217 expression levels in the tumor and normal tissues of the CRC patients were compared, there was a statistically slightly significant increase in miR-217 expression in the tumor tissues. When the serum and the tumor tissue samples of the patients were compared regarding miR-217 expression, a higher expression level was observed in the tumor tissues. There was no difference between the serum samples of the patients and healthy individuals in terms of miR-217 expression levels. In conclusion, it is thought that the increased expression of miR-217 in tumor tissue samples of the patients may indicate its oncogenic role in CRC development.

$\mathrm{K}$-ras is one of the recommended biomarkers for CRC (29). You et al, demonstrated that when the K-ras protein level was 
higher in CRC tumor tissues compared to normal tissues, its mRNA level was altered irregularly (30). In our study, when K-ras expression levels were examined between tumor tissue and non-tumor adjacent tissue samples of the patients, we observed higher K-ras levels in tumor tissues. Studies have shown that miR-181d and miR-217 directly target the K-ras gene (31-33).

One of the limitations of this study is the relatively small size of the study population. Another limitation is that we could not use any other techniques such as western blotting to confirm the expression analysis.

According to our findings, we think that expression levels of miR-181d and miR-217 are related to the increased expression of $\mathrm{K}$-ras gene in tumor tissues and that the expression of K-ras gene, which plays an oncogenic role in colorectal cancer development, is regulated by these miRNAs. It can be concluded that miR-181d and miR-217 may function as oncogenic miRNAs in the development of colorectal cancer.

\section{References}

1. Jaiswal AS, Balusu R, Narayan S. Involvement of adenomatous polyposis coli in colorectal tumorigenesis. Front Biosci 2005; 10: 1118-1134.

2. Zhang L, Shay JW. Multiple Roles of APC and its Therapeutic Implications in Colorectal Cancer. J Natl Cancer Inst 2017; 109 (8).

3. Half E, Bercovich D, Rozen P. Familial adenomatous polyposis. Orphanet J Rare Dis 2009; 4:22.

4. Kwong LN, Dove WF. APC and its modifiers in colon cancer. Adv Exp Med Biol 2009; 656: 85-106.

5. Alberici P, Fodde R. The role of the APC tumor suppressor in chromosomal instability. Genome Dyn 2006; 1: 149-170.

6. Arvelo F, Sojo F, Cotte C. Biology of colorectal cancer. Ecancermedicalscience 2015; 9: 520.

7. Sameer AS. Colorectal cancer: molecular mutations and polymorphisms. Front Oncol 2013; 3: 114.

8. Ling H, Fabbri M, Calin GA. MicroRNAs and other non-coding RNAs as targets for anticancer drug development. Nat Rev Drug Discov 2013; 12 (11): 847-865.

9. Bandyopadhyay S, Mitra R, Maulik U, Zhang MQ. Development of the human cancer microRNA network. Silence 2010; 1 (1): 6.

10. Wang J, Zhang KY, Liu SM, Sen S. Tumor-associated circulating microRNAs as biomarkers of cancer. Molecules 2014; 19 (2): 1912-1938.

11. Lan H, Lu H, Wang X, Jin H. MicroRNAs as potential biomarkers in cancer: opportunities and challenges. Biomed Res Int 2015; 2015: 125094.

12. Hwang HW, Mendell JT. MicroRNAs in cell proliferation, cell death, and tumorigenesis. Br J Cancer 2006; 94 (6): 776-780.

13. Jansson MD, Lund AH. MicroRNA and cancer. Mol Oncol 2012; 6 (6): 590-610.

14. Colussi D, Brandi G, Bazzoli F, Ricciardiello L. Molecular pathways involved in colorectal cancer: implications for disease behavior and prevention. Int J Mol Sci 2013; 14 (8): 16365-16385.

15. Li Y, Li J, Sun X, Chen J, Sun X, Zheng J et al. MicroRNA-27a functions as a tumor suppressor in renal cell carcinoma by targeting epidermal growth factor receptor. Oncol Lett 2016; 11 (6): 4217-4223.

16. Myatt SS, Wang J, Monteiro LJ, Christian M, Ho KK, Fusi L et al. Definition of microRNAs that repress expression of the tumor suppressor gene FOXO1 in endometrial cancer. Cancer Res 2010; 70 (1): 367-377.
17. Liang S, Zhang N, Deng Y, Chen L, Zhang $Y$, Zheng Z et al. Increased Serum Level of MicroRNA-663 Is Correlated with Poor Prognosis of Patients with Nasopharyngeal Carcinoma. Dis Markers 2016; 2016: 7648215.

18. Liang S, Zhang N, Deng Y, Chen L, Zhang Y, Zheng Z et al. miR-663 promotes NPC cell proliferation by directly targeting CDKN2A. Mol Med Rep 2017; 16 (4): 4863-4870.

19. Liu ZY, Zhang GL, Wang MM, Xiong YN, Cui HQ. MicroRNA-663 targets TGFB1 and regulates lung cancer proliferation. Asian Pac J Cancer Prev 2011; 12 (11): 2819-2823.

20. Wang N, Zeng L, Li Z, Zhen Y, Chen H. Serum miR-663 expression and the diagnostic value in colorectal cancer. Artif Cells Nanomed Biotechnol 2019; 47 (1): 2650-2653.

21. Park MG, Kim JS, Park SY, Lee SA, Kim HJ, Kim CS et al. MicroRNA-27 promotes the differentiation of odontoblastic cell by targeting APC and activating Wnt/beta-catenin signaling. Gene 2014; 538 (2): 266-272.

22. Zhang Z, Liu S, Shi R, Zhao G. miR-27 promotes human gastric cancer cell metastasis by inducing epithelial-to-mesenchymal transition. Cancer Genet 2011; 204 (9): 486-491.

23. Kim JS, Park MG, Lee SA, Park SY, Kim HJ, Yu SK et al. Downregulation of adenomatous polyposis coli by microRNA-663 promotes odontogenic differentiation through activation of Wnt/beta-catenin signaling. Biochem Biophys Res Commun 2014; 446 (4): 894-900.

24. Miao CG, Shi WJ, Xiong YY, Yu H, Zhang XL, Qin MS et al. MicroRNA-663 activates the canonical Wnt signaling through the adenomatous polyposis coli suppression. Immunol Lett 2015; 166 (1): 45-54.

25. Guo X, Zhu Y, Hong X, Zhang M, Qiu X, Wang Z et al. miR-181d and c-myc-mediated inhibition of CRY2 and FBXL3 reprograms metabolism in colorectal cancer. Cell Death Dis 2017; 8 (7): e2958.

26. Huang L, Wen C, Yang $X$, Lou Q, Wang $X$, Che $J$ et al. PEAK1, acting as a tumor promoter in colorectal cancer, is regulated by the EGFR/KRas signaling axis and miR-181d. Cell Death Dis 2018; 9 (3): 271.

27. Zhang Q, Yuan Y, Cui J, Xiao T, Jiang D. MiR-217 Promotes Tumor Proliferation in Breast Cancer via Targeting DACH1. J Cancer 2015; 6 (2): 184-191.

28. Vychytilova-Faltejskova P, Kiss I, Klusova S, Hlavsa J, Prochazka V, Kala Z et al. MiR-21, miR-34a, miR-198 and miR-217 as diagnostic and prognostic biomarkers for chronic pancreatitis and pancreatic ductal adenocarcinoma. Diagn Pathol 2015; 10: 38.

29. Ugorcakova J, Hlavaty T, Novotna T, Bukovska G. Detection of point mutations in KRAS oncogene by real-time PCR-based genotyping assay in GIT diseases. Bratisl Lek Listy. 2012; 113 (2): 73-79.

30. You C, Liang H, Sun W, Li J, Liu Y, Fan Q et al. Deregulation of the miR-16-KRAS axis promotes colorectal cancer. Sci Reports 2016; 6: 37459.

31. Guo J, Feng Z, Huang Z, Wang H, Lu W. MicroRNA-217 functions as a tumour suppressor gene and correlates with cell resistance to cisplatin in lung cancer. Mol Cells 2014; 37 (9): 664-671.

32. Wang XF, Shi ZM, Wang XR, Cao L, Wang YY, Zhang JX et al. MiR-181d acts as a tumor suppressor in glioma by targeting K-ras and Bcl2. J Cancer Res Clin Oncol 2012; 138 (4): 573-584.

33. Zhao WG, Yu SN, Lu ZH, Ma YH, Gu YM, Chen J. The miR-217 microRNA functions as a potential tumor suppressor in pancreatic ductal adenocarcinoma by targeting KRAS. Carcinogenesis 2010; 31 (10): 1726-1733.

Received March 10, 2020. Accepted April 1, 2020. 